\title{
Lung cancer screening in patients with chronic obstructive pulmonary disease
}

\author{
Jessica Gonzalez ${ }^{1}$, Marta Marín ${ }^{1}$, Pablo Sánchez-Salcedo ${ }^{2}$, Javier J. Zulueta ${ }^{1}$ \\ ${ }^{1}$ Respiratory Medicine Service, Clinica Universidad de Navarra, Pamplona, Spain; ${ }^{2}$ Respiratory Medicine Service, Complejo Hospitalario de Navarra, \\ Pamplona, Spain \\ Contributions: (I) Conception and design: All authors; (II) Administrative support: JJ Zulueta, P Sánchez-Salcedo; (III) Provision of study materials or \\ patients: All authors; (IV) Collection and assembly of data: All authors; (V) Data analysis and interpretation: All authors; (VI) Manuscript writing: All \\ authors; (VII) Final approval of manuscript: All authors. \\ Correspondence to: Javier J. Zulueta. Respiratory Medicine Service, Clinica Universidad de Navarra, Av. Pio XII s/n, Pamplona 31008 , Spain. \\ Email: jzulueta@unav.es.
}

\begin{abstract}
Lung cancer and chronic obstructive pulmonary disease (COPD) are two intimately related diseases, with great impact on public health. Annual screening using low-dose computed tomography (LDCT) of the chest significantly reduces mortality due to lung cancer, and several scientific societies now recommend this technique. COPD, defined by the presence of airflow obstruction [forced expiratory volume and forced vital capacity (FVC) ratio less than 0.70 ], and their clinical phenotypes, namely emphysema and chronic bronchitis, have been associated with increased lung cancer risk. Several epidemiological studies, including lung cancer screening trials, have found a 2- to 4-fold increase in lung cancer risk in patients with COPD when compared to individuals without airflow obstruction. Part of the risk attributed to airflow obstruction appears to be derived from the presence of radiographic emphysema. The latter has proven to be an important lung cancer risk factor in smokers without airflow obstruction and even in never smokers. This evidence supports the idea of including patients with COPD and/or emphysema in lung cancer screening programs. There is evidence that lung cancer screening in this population is effective and can potentially reduce mortality. Specific lung cancer risk scores have been developed for patients with COPD [COPD lung cancer screening score (LUCSS) and COPD-LUCSS-diffusing capacity for carbon monoxide (DLCO)] to identify those at high risk. A multidisciplinary approach for an adequate patient selection, especially of patients with severe disease, is key to maximize benefits and reduce harms from lung cancer screening in this population. Patients with COPD included in lung cancer screening programs could also benefit from other interventions, such as smoking cessation and adequate treatment.
\end{abstract}

Keywords: Lung cancer screening; chronic obstructive pulmonary disease (COPD); emphysema; lung cancer

Submitted Feb 24, 2016. Accepted for publication Mar 28, 2016.

doi: $10.21037 /$ atm.2016.03.57

View this article at: http://dx.doi.org/10.21037/atm.2016.03.57

\section{Introduction}

Lung cancer and chronic obstructive pulmonary disease (COPD) are two major public health problems. Data from the Global Burden of Disease Study 2013 states that lung cancer and COPD are among the six non-communicable leading causes of years of life lost globally, especially in developed and high-income countries (1). Lung cancer is the leading cause of death from malignant disease worldwide, with more deaths from lung cancer than from colon, prostate and breast cancer together $(2,3)$. It is estimated that by 2030 it will still be one of the main causes of death due to the fact that lung cancer diagnosis is usually made at an advanced stage (4).

COPD is the leading cause of death among chronic respiratory diseases, causing 6 times more deaths than asthma or interstitial lung diseases (1). This number could be even higher owed to the fact that COPD is often 
an underdiagnosed disease (5). In almost every country lung cancer is within the top leading causes of death and COPD is closely positioned (1). This is no coincidence, as several epidemiological studies have observed an important association between these two diseases and various mechanisms to explain this relationship has been proposed. Chronic inflammation due to tobacco exposure and lung repair mechanisms in COPD patients appear to be key features for lung cancer development (6).

Lung cancer screening with low-dose computed tomography (LDCT) of the chest has emerged in the las few years as a technique that could improve the burden of lung cancer. The International Early Lung Cancer Action Program (I-ELCAP) demonstrated that using LDCT to screen for lung cancer following a standardized protocol could detect up to $85 \%$ of lung cancers in clinical stage I (7). Moreover, the National Lung Screening Trial (NLST) further demonstrated that lung cancer screening with LDCT significantly reduces lung cancer mortality (8). Both studies highlight the value of lung cancer screening and served as fundamental evidence for the positive recommendation for screening issued by the United States Preventive Services Task Force (USPSTF) (9).

Screening programs are most effective when high risk populations are targeted. There is plenty of evidence that points to COPD as an important risk factor for lung cancer (10-14). In this review we present the current available evidence linking both diseases as well as the role of lung cancer screening in this high-risk population.

\section{Physiopathology and mechanisms}

Various mechanisms have been proposed to explain the association between COPD and lung cancer. These mechanisms include genetic susceptibility, deoxyribonucleic acid (DNA) damage and repair, epigenetics, downregulation of specific microRNA, expression of pro-inflammatory genes induced by hypoxia, tumor growth factor-B and integrins, telomere length and dysfunction, and immune adaptive responses (15-19). There is no definitive explanation for the association, and interactions between many of them are presumably present. It is not the purpose of this review to thoroughly discuss these mechanisms, and we suggest the reader to address the appropriate sources.

\section{COPD, emphysema, and lung cancer risk}

The association between COPD and lung cancer was first described in the 1980's by Skillrud et al. (10) and Tockman et al. (11), when a 4-fold increase in lung cancer incidence and mortality, respectively, was described in patients with airflow obstruction. Since then, many cohort studies, including lung cancer screening trials, have demonstrated that patients with COPD have 2-4 times greater risk of incident lung cancer when compared to those who do not have COPD (12-14,20). This greater risk prevails even after controlling for smoking exposure. The timing of COPD diagnosis also appears to be important. In a sample of almost 12,000 incident lung cancer cases, where $23 \%$ had a prior diagnosis of COPD, the risk of lung cancer among patients diagnosed of COPD within 6 months of the lung cancer diagnosis was 2-3 times higher than those diagnosed of COPD more than one and more than ten years before, respectively (21).

Several studies have assessed the risk between the degree of airflow obstruction and lung cancer, but results have been contradictive. Using data from the Body mass index, Airflow obstruction, Dyspnea, Exercise performance (BODE) observational cohort of patients with COPD, de Torres et al. described a higher risk of incident lung cancer in patients with mild and moderate airflow obstruction (HR 3.05; 95\% CI: 1.41-6.59, and HR 2.06; 95\% CI: 1.01-4.18, respectively) (22). Similarly, in the Pamplona subcohort of the International Early Lung Cancer Detection Program (P-IELCAP), 94\% of the patients with COPD diagnosed with lung cancer had spirometric global initiative for chronic obstructive lung disease (GOLD) grades 1 and 2 (23). These results contrast with those from other studies. In the Pittsburgh Lung Screening Study (PLuSS) (13) and in the National Health and Nutrition Examination Survey (24), the risk for incident lung cancer was almost 3-fold greater in patients with moderate to very severe airflow obstruction as compared to patients with mild COPD.

The association between impaired lung function and the risk of lung cancer has been confirmed in a recent meta-analysis. Compared with the highest quintile of forced expiratory volume in one second (FEV1 $>100 \%$ predicted), the lowest quintile of FEV1 (< 70\% predicted) was associated with a 2- and almost 4-fold increase in lung cancer risk in men and women, respectively (25). In patients with COPD, a reduced FEV1, as small as $90 \%$ predicted, was associated with more than 2 -fold increase in lung cancer risk (26).

The evidence presented so far has focused on spirometric defined COPD, i.e., FEV1 and forced vital capacity (FVC) ratio less than $70 \%$, and includes individuals who 
have chronic bronchitis and/or emphysema. The use of computed tomography has allowed physicians to assess the presence emphysema in patients with COPD. de-Torres and colleagues analyzed the impact of emphysema presence on LDCT on lung cancer risk (12). Lung cancer incidence density in individuals with emphysema was 3 times higher than in those without emphysema. Even in patients without airflow obstruction, the presence of emphysema increased the risk of incident lung cancer 4-fold (RR 4.33; 95\% CI: 1.04-18.16) (12). Interestingly, when airflow obstruction and emphysema were included in a single regression model, only emphysema remained as an independent risk factor for lung cancer, suggesting that part of the risk attributed to spirometric defined COPD could be due to the presence of emphysema. In PLuSS, Wilson and colleagues obtained the same results (13).

The importance of emphysema has also been assessed in non-smoking populations. In a population of almost 500,000 nonsmokers who were followed for a 20 -year period, lung cancer mortality was significantly associated with emphysema (HR 1.66; 95\% CI: 1.06-2.59) (27). Similarly, the I-ELCAP group compared the impact of emphysema on lung cancer prevalence in current, former and never smokers who underwent lung cancer screening (28). A surprising fact of this study was that lung cancer prevalence in smokers (current or former) with emphysema was similar to that found in never smokers with emphysema ( $2.1 \%$ and $2.6 \%$, respectively, $\mathrm{P}=0.61)$. The odds ratio for lung cancer prevalence in nonsmokers with emphysema was 6-fold greater when compared to nonsmokers without emphysema (28).

Evidence regarding the severity of emphysema and lung cancer risk is not consistent. In PLuSS, Wilson and colleagues found that lung cancer risk was higher for individuals with mild emphysema, followed by those who had moderate-severe, and traces of emphysema, respectively (13). Similarly, Li and colleagues (29) found that lung cancer risk did not increase with more extense empshyema (OR 3.33; 95\% CI: 2.30-4.82, and OR 3.80; $95 \%$ CI: $2.78-5.19$, for $\geq 10 \%$ and $\geq 5 \%$ emphysema, respectively). On the other hand, Zulueta et al. did find a linear trend between emphysema severity and lung cancer mortality, but the association was only significant for marked emphysema (30). These results contrasts to those from Maldonado et al. (14) and Kishi et al. (31) where no significant associations between emphysema and lung cancer risk were found. A possible explanation for these contradictive results can be found in the methodology used to determine the presence and the amount of emphysema. While the latter two studies used automated emphysema quantification, the first two relied on visually assessed emphysema. In this regard, in a meta-analysis by Smith et al., only visually determined emphysema on CT was independently associated with an increased lung cancer risk (32).

The presence of emphysema around the region of a tumor has also been subject of study. Studies coincide in the fact that lung cancer develops more often in areas of the lung with more emphysema. Bishawi and co-workers found a strong association for cancer being located in the area with the highest degree of emphysema, usually being the right upper quadrant (33). Using data from the Lung Tissue Research Consortium, Hohberger and colleagues found that the odds of having a more severe emphysema score in the location of lung cancer was 1.34 (95\% CI: 1.11-1.62), once again, more frequently in the right upper lobe (34). In a more recent study, Kinsey and colleagues used an automated densitometric analysis for the measurement of emphysema (Airway Inspector Software) to assess whether emphysema in the region where the tumor occurs results in larger tumors (35). The mean difference in tumor diameter between the lower and upper quartiles of emphysema was $0.7 \mathrm{~cm}(21 \%)$, and increasing emphysema in the region of the tumor was significantly associated with a worse overall survival (for each $10 \%$ increase in peritumoral emphysema score, there was a $30 \%$ increase in the hazard of death).

It is worth saying that the risk of lung cancer is the greatest when both airflow obstruction and emphysema coexist. Lung cancer incidence density per 1,000 personyears of follow-up was almost 2-fold greater in participants with both diseases when compared to participants who had either one of them (12). In PLuSS, for a same degree of airflow obstruction, the presence of emphysema increased the odds ratio of having lung cancer (13).

Finally, the association between chronic bronchitis and lung cancer has also been assessed. Pooled analyses from the International Lung Cancer Consortium (36) and the SYNERGY Project (consortium of international lung cancer case-control studies) (37), have described significant associations between chronic bronchitis and lung cancer. In the former, based on data from 13 studies, a previous diagnosis of chronic bronchitis was associated with a $47 \%$ increase in lung cancer risk overall (RR 1.47; 95\% CI: 1.29 1.68). The risk was slightly higer in ever smokers (RR 1.63; 95\% CI: 1.40-1.89) (36). In the latter study, this association was only significant in males (OR 1.33 ; 95\% CI 1.20-1.48 
vs. OR 1.12; 95\% CI: 0.92-1.35 in women) (37).

The evidence so far shows significant associations between lung cancer and spirometric defined COPD, including each of their classical phenotypes, such as chronic bronchitis and emphysema. Based on the different risk assessments, emphysema and airflow obstruction clearly stand out.

\section{Airflow obstruction and emphysema as selection criteria for lung cancer screening}

The significant reduction in lung cancer mortality observed in the NLST trial provided enough evidence for different scientific societies to make recommendations in favor of lung cancer screening with LDCT, including the USPSTF (9,38-43). All these societies mainly rely on the entry criteria used in the NLST trial: men and women between 55 and 74 years of age, currently smoking or having quit within the previous 15 years, and with a smoking history of at least 30 pack-years (8). However, there is evidence that these criteria might not be sensitive enough in detecting lung cancer cases. In some lung cancer representative samples, more than $50 \%$ of lung cancer cases didn't meet NLST criteria, otherwise meaning that they wouldn't have been included in a lung cancer screening program (44-47). Wang and colleagues described the trend of the proportion of patients with lung cancer meeting USPSTF screening criteria (same as NLST with upper limit for age extended to 80 years) between 1984 and 2011. Out of 1351 lung cancer cases, the proportion of individuals who met USPSTF selection criteria decreased from $56.8 \%$ in $1984-1990$ to $43.3 \%$ in 2005-2011 (47). If we keep using these entry criteria and these trends continue its descent, we will have to deal with more lung cancers outside of a screening setting. These data suggest that we need to find additional selection criteria to identify more individuals at risk. Considering the vast evidence pointing at COPD and emphysema as important risk factors for lung cancer, it isn't strange that these criteria are being suggested as selection criteria.

In this sense, a few medical societies have included additional risk factors in addition to the NLST/USPSTF criteria. The American Association for Thoracic Surgery (39) and the National Comprehensive Cancer Network (40) guidelines both give initial recommendations using USPSTF and NLST entry criteria, respectively. However, in a secondary recommendation, they include younger individuals with less smoking exposure ( $\geq 50$ years of age and $\geq 20$ pack/year smoking history) provided they have one additional risk factor, such as COPD.

In line with this, our group has proposed complementing NLST criteria with visually determined emphysema (46). In P-IELCAP and PLuSS, both lung cancer screening cohorts with broader entry criteria than NLST, only $36 \%$ and $59 \%$ of their participants, respectively, met NLST entry criteria. This resulted in a $39 \%$ and $20 \%$ reduction in the lung cancer diagnostic yield, respectively. By using emphysema as a complementary criterion to NLST (NLST/E) for annual screening, only three incident lung cancers would have been left undetected in each cohort, representing a 3\% and $12 \%$ reduction in diagnostic yield in PLuSS and P-IELCAP, respectively. Furthermore, annual lung cancer detection rates and the number of individuals needed to be screened in one year to detect one lung cancer (NND) were higher in the NLST/E groups, when compared to NLST alone, highlighting the importance of emphysema in selecting individuals at high risk (46). In the Continuous Observation of Smoking Subjects (COSMOS) lung cancer screening trial, different models to stratify the risk of developing lung cancer during annual screening rounds were developed. When compared to other LDCT findings, such as nodule size, nodule type (solid vs. non-solid), and lung function, emphysema was a much stronger lung cancer predictor in most of the models tested (48).

Using the NLST population sample, Kovalchik and colleagues built a lung cancer death risk prediction model to test whether screening benefits and harms vary according to lung cancer risk (49). They observed that the greatest numbers of deaths from lung cancer were prevented in the group that was at greatest risk of death. In the risk model developed, emphysema was the factor with the greatest risk of death (HR 1.56; 95\% CI: 1.20-2.04). They concluded that a risk-based strategy for lung cancer screening could provide a rational, empirical framework for the inclusion of NLST-ineligible smokers at high risk of lung cancer death, with whom we certainly agree (49).

Recently, a lung cancer screening score (LUCSS) to identify patients with COPD with the highest risk of developing lung cancer (COPD-LUCSS) was developed by our group (50). Age, body mass index (BMI), packyears of smoking history, and the presence of radiological emphysema were included in the score (Table 1). Patients were classified into two categories depending on their total score: low-risk (0-6 points) or high risk ( $\geq 7$ points). The latter group had a 3 -fold increase in lung cancer risk when compared to the low-risk group (HR 3.5; 95\% CI: 1.7-7.1) (50). Considering that in clinical practice most 
Table 1 COPD lung cancer screening score (LUCSS)

\begin{tabular}{lc}
\hline Variable & Points assigned \\
\hline $\mathrm{BMI}<25 \mathrm{~kg} / \mathrm{m}^{2}$ & 1 \\
Pack-years $>60$ & 2 \\
Age $>60$ years & 3 \\
Emphysema presence on LDCT & 4 \\
Total & 10 \\
\hline
\end{tabular}

BMI, body mass index; LDCT, low-dose computed tomography; COPD, chronic obstructive pulmonary disease.

Table 2 Modified COPD lung cancer screening score (LUCSS) DLCO

\begin{tabular}{lc}
\hline Variable & Points assigned \\
\hline $\mathrm{BMI}<25 \mathrm{~kg} / \mathrm{m}^{2}$ & 1.5 \\
Pack-years $>60$ & 1 \\
Age $>60$ years & 2.5 \\
Emphysema presence on LDCT & 3 \\
Total & 8 \\
\hline
\end{tabular}

BMI, body mass index; LDCT, low-dose computed tomography; DLCO, diffusing capacity for carbon monoxide; COPD, chronic obstructive pulmonary disease.

patients with COPD do not have a CT available, and thus, no way of confirming emphysema, the same authors later reported a modified version of the score, where diffusing capacity for carbon monoxide (DLCO) was used instead of visually detected emphysema (Table 2). In this new score (COPD-LUCSS-DLCO), participants were also classified into low (0-3 points) and high risk groups (3.5-8 points), where the latter had a 2.4 increased risk of death from lung cancer when compared to the low-risk group (95\% CI: 2.0 2.7) (51). These results do not mean patients with COPD in the low-risk category should be left aside and not included in screening programs, as they still have a higher risk of developing lung cancer than healthy smokers. Although it is not completely established how each risk category should be managed, it is possible that individuals at risk for lung cancer may benefit from different screening regimes, with less intense screening for lower-risk groups. This may have a significant impact on costs of lung cancer screening.

\section{Clinical considerations}

There are concerns about the inclusion of patients with
COPD and/or emphysema in lung cancer screening programs, as the potential benefits of screening might be surpassed by the increased risk of death inherent to COPD and/or emphysema (especially if airflow obstruction or emphysema are severe) and their associated comorbidities $(52,53)$. de-Torres and colleagues explored the impact of screening in a sample of patients with mild and moderate COPD from a lung cancer screening trial, based on preliminary data from their group showing that in these subgroups is where lung cancer risk is greater (54). By comparing lung cancer mortality in this group with that of a matched sample of COPD patients who didn't undergo screening, they observed that the mortality incidence density was 30-times lower in the group that underwent screening (0.08 vs. 2.48 deaths per 100-person-years; $\mathrm{P}=0.001)$. In the Danish Lung Cancer Screening Trial (DLCST), although no statistically significant effects of CT screening on lung cancer mortality were found overall, non-significant trends were found among patients with COPD, where a $24 \%$ relative reduction in the risk of death was found in the group who underwent screening (55). In the subgroup of participants from the NLST who had a spirometry done, Young and colleagues observed that the lung cancers that developed in patients with COPD appeared to be more aggressive, and less overdiagnosis was seen in this group when compared to lung cancers in participants with no airflow obstruction (56).

In terms of treatments, there is growing evidence that newer surgical treatments, such as sublobar resections or lung volume reduction surgeries, or ablative treatments, such as stereotactic body radiotherapy, offer good long-term outcomes in patients with advanced age, as well as advanced COPD and/or emphysema (57-66). In any case, adequate patient selection is key to reduce screening's potential harms to a minimum. The implementation of a multidisciplinary approach and registry monitoring as recommended by guidelines will help overcome this potential problem.

Patients with COPD and/or emphysema enrolled in lung screening programs could also benefit from smoking cessation treatments. In the DLCST, authors found and overall increasing smoking cessation rate during the screening program, where higher motivation to quit at baseline predicted smoking abstinence at the final screening round (67). Positive results in the LDCT are especially associated with increased abstinence (68). Achieving smoking abstinence in patients with COPD would be especially useful, as it is the most effective intervention in stopping the progression of the disease, as well as increasing 
survival and reducing morbidity, including lung cancer risk (69). If spirometry, a simple, inexpensive and validated diagnostic test, were to be added to lung cancer screening programs, smokers being screened could also benefit from early diagnosis of COPD, a disease overwhelmingly underdiagnosed (5). Presently, screening for COPD is not recommended since there is no known benefit to treating asymptomatic patients. However, we can now have a great impact on mortality rates in patients with COPD by detecting lung cancer, one of the main causes of death in this population, in early, curable stages.

\section{Conclusions}

Airflow obstruction and emphysema are important risk factors for lung cancer, with significant associations not only with lung cancer incidence, but with death from lung cancer. There is evidence that lung cancer screening in patients with COPD is effective, and newer, less aggressive treatments offer new opportunities to patients with advanced disease, who otherwise wouldn't be included in screening programs or offered potential curative treatments. The COPDLUCSS and the COPD-LUCSS-DLCO are useful tools to identify patients with COPD with high and low risk of lung cancer, although their specific role in screening programs is yet to be determined. A multidisciplinary assessment is essential to include patients with COPD in lung cancer screening programs, especially for those with advanced disease and associated comorbidities, in order to reduce potential harms from diagnostic and therapeutic procedures. Besides improved early lung cancer detection, patients with COPD included in screening programs could benefit from smoking cessation interventions, complete characterization of their disease, and adequate treatment. Finally, lung cancer screening programs offer a great opportunity to uncover the vast underdiagnosis of COPD.

\section{Acknowledgements}

None.

\section{Footnote}

Conflicts of Interest: The above manuscript hasn't been published or submitted elsewhere. Dr. Zulueta is a non-paid member of the Medical Advisory Board and is a shareholder of VisionGate, Inc. The authors have no conflicts of interest to declare.

\section{References}

1. GBD 2013 Mortality and Causes of Death Collaborators. Global, regional, and national age-sex specific all-cause and cause-specific mortality for 240 causes of death, 1990-2013: a systematic analysis for the Global Burden of Disease Study 2013. Lancet 2015;385:117-71.

2. Spiro SG, Silvestri GA. One hundred years of lung cancer. Am J Respir Crit Care Med. 2005;172:523-9.

3. Shlomi D, Ben-Avi R, Balmor GR, et al. Screening for lung cancer: time for large-scale screening by chest computed tomography. Eur Respir J 2014;44:217-38.

4. Mathers CD, Loncar D. Projections of global mortality and burden of disease from 2002 to 2030. PLoS Med 2006;3:e442.

5. Lamprecht B, Soriano JB, Studnicka M, et al. Determinants of underdiagnosis of COPD in national and international surveys. Chest 2015;148:971-85.

6. Houghton AM, Mouded M, Shapiro SD. Common origins of lung cancer and COPD. Nat Med 2008;14:1023-4.

7. International Early Lung Cancer Action Program Investigators, Henschke CI, Yankelevitz DF, et al. Survival of patients with stage I lung cancer detected on CT screening. N Engl J Med 2006;355:1763-71.

8. National Lung Screening Trial Research Team, Aberle DR, Adams AM, et al. Reduced lung-cancer mortality with low-dose computed tomographic screening. N Engl J Med 2011;365:395-409.

9. Moyer VA; U.S. Preventive Services Task Force. Screening for lung cancer: U.S. Preventive Services Task Force recommendation statement. Ann Intern Med 2014;160:330-8.

10. Skillrud DM, Offord KP, Miller RD. Higher risk of lung cancer in chronic obstructive pulmonary disease. A prospective, matched, controlled study. Ann Intern Med 1986;105:503-7.

11. Tockman MS, Anthonisen NR, Wright EC, et al. Airways obstruction and the risk for lung cancer. Ann Intern Med 1987;106:512-8.

12. de Torres JP, Bastarrika G, Wisnivesky JP, et al. Assessing the relationship between lung cancer risk and emphysema detected on low-dose CT of the chest. Chest 2007;132:1932-8.

13. Wilson DO, Weissfeld JL, Balkan A, et al. Association of radiographic emphysema and airflow obstruction with lung cancer. Am J Respir Crit Care Med 2008;178:738-44.

14. Maldonado F, Bartholmai BJ, Swensen SJ, et al. Are airflow obstruction and radiographic evidence of emphysema risk 
factors for lung cancer? A nested case-control study using quantitative emphysema analysis. Chest 2010;138:1295-302.

15. Durham AL, Adcock IM. The relationship between COPD and lung cancer. Lung Cancer 2015;90:121-7.

16. Houghton AM. Mechanistic links between COPD and lung cancer. Nat Rev Cancer 2013;13:233-45.

17. Rooney C, Sethi T. The epithelial cell and lung cancer: the link between chronic obstructive pulmonary disease and lung cancer. Respiration 2011;81:89-104.

18. Adcock IM, Caramori G, Barnes PJ. Chronic obstructive pulmonary disease and lung cancer: new molecular insights. Respiration 2011;81:265-84.

19. Caramori G, Casolari P, Cavallesco GN, et al. Mechanisms involved in lung cancer development in COPD. Int J Biochem Cell Biol 2011;43:1030-44.

20. Lowry KP, Gazelle GS, Gilmore ME, et al. Personalizing annual lung cancer screening for patients with chronic obstructive pulmonary disease: A decision analysis. Cancer 2015;121:1556-62.

21. Powell HA, Iyen-Omofoman B, Baldwin DR, et al. Chronic obstructive pulmonary disease and risk of lung cancer: the importance of smoking and timing of diagnosis. J Thorac Oncol 2013;8:6-11.

22. de Torres JP, Marín JM, Casanova C, et al. Lung cancer in patients with chronic obstructive pulmonary disease-incidence and predicting factors. Am J Respir Crit Care Med 2011;184:913-9.

23. Sanchez-Salcedo P, Berto J, de-Torres JP, et al. Lung cancer screening: fourteen year experience of the Pamplona early detection program (P-IELCAP). Arch Bronconeumol 2015;51:169-76.

24. Mannino DM, Aguayo SM, Petty TL, et al. Low lung function and incident lung cancer in the United States: data From the First National Health and Nutrition Examination Survey follow-up. Arch Intern Med 2003;163:1475-80.

25. Wasswa-Kintu S, Gan WQ, Man SF, et al. Relationship between reduced forced expiratory volume in one second and the risk of lung cancer: a systematic review and metaanalysis. Thorax 2005;60:570-5.

26. Calabrò E, Randi G, La Vecchia C, et al. Lung function predicts lung cancer risk in smokers: a tool for targeting screening programmes. Eur Respir J 2010;35:146-51.

27. Turner MC, Chen Y, Krewski D, et al. Chronic obstructive pulmonary disease is associated with lung cancer mortality in a prospective study of never smokers. Am J Respir Crit Care Med 2007;176:285-90.

28. Henschke CI, Yip R, Boffetta P, et al. CT screening for lung cancer: Importance of emphysema for never smokers and smokers. Lung Cancer 2015;88:42-7.

29. Li Y, Swensen SJ, Karabekmez LG, et al. Effect of emphysema on lung cancer risk in smokers: a computed tomography-based assessment. Cancer Prev Res (Phila) 2011;4:43-50.

30. Zulueta JJ, Wisnivesky JP, Henschke CI, et al. Emphysema scores predict death from COPD and lung cancer. Chest 2012;141:1216-23.

31. Kishi K, Gurney JW, Schroeder DR, et al. The correlation of emphysema or airway obstruction with the risk of lung cancer: a matched case-controlled study. Eur Respir J 2002;19:1093-8.

32. Smith BM, Pinto L, Ezer N, et al. Emphysema detected on computed tomography and risk of lung cancer: a systematic review and meta-analysis. Lung Cancer 2012;77:58-63.

33. Bishawi M, Moore W, Bilfinger T. Severity of emphysema predicts location of lung cancer and 5-y survival of patients with stage I non-small cell lung cancer. J Surg Res 2013;184:1-5.

34. Hohberger LA, Schroeder DR, Bartholmai BJ, et al. Correlation of regional emphysema and lung cancer: a lung tissue research consortium-based study. J Thorac Oncol 2014;9:639-45.

35. Kinsey CM, San José Estépar R, Wei Y, et al. Regional Emphysema of a Non-Small Cell Tumor Is Associated with Larger Tumors and Decreased Survival Rates. Ann Am Thorac Soc 2015;12:1197-205.

36. Brenner DR, Boffetta P, Duell EJ, et al. Previous lung diseases and lung cancer risk: a pooled analysis from the International Lung Cancer Consortium. Am J Epidemiol 2012;176:573-85.

37. Denholm R, Schüz J, Straif K, et al. Is previous respiratory disease a risk factor for lung cancer? Am J Respir Crit Care Med 2014;190:549-59.

38. Kauczor HU, Bonomo L, Gaga M, et al. ESR/ERS white paper on lung cancer screening. Eur Respir J 2015;46:28-39.

39. Jaklitsch MT, Jacobson FL, Austin JH, et al. The American Association for Thoracic Surgery guidelines for lung cancer screening using low-dose computed tomography scans for lung cancer survivors and other high-risk groups. J Thorac Cardiovasc Surg 2012;144:33-8.

40. Wood DE, Eapen GA, Ettinger DS, et al. Lung cancer screening. J Natl Compr Canc Netw 2012;10:240-65.

41. Wender R, Fontham ET, Barrera E Jr, et al. American Cancer Society lung cancer screening guidelines. CA Cancer J Clin 2013;63:107-17. 
42. Detterbeck FC, Mazzone PJ, Naidich DP, et al. Screening for lung cancer: Diagnosis and management of lung cancer, 3rd ed: American College of Chest Physicians evidencebased clinical practice guidelines. Chest 2013;143:e78S-92S.

43. American Lung Association. Providing guidance on lung cancer screening to patients and physicians. Available online: http://www. lung.org/lung-disease/lung-cancer/ lung-cancer-screening- guidelines/lung-cancer-screening. pdf, accessed Oct 17, 2013.

44. Pinsky PF, Berg CD. Applying the National Lung Screening Trial eligibility criteria to the US population: what percent of the population and of incident lung cancers would be covered? J Med Screen 2012;19:154-6.

45. Young RP, Hopkins RJ. Lung cancer risk prediction to select smokers for screening CT--letter. Cancer Prev Res (Phila) 2012;5:697-8; author reply 699.

46. Sanchez-Salcedo P, Wilson DO, de-Torres JP, et al. Improving selection criteria for lung cancer screening. The potential role of emphysema. Am J Respir Crit Care Med 2015;191:924-31.

47. Wang Y, Midthun DE, Wampfler JA, et al. Trends in the proportion of patients with lung cancer meeting screening criteria. JAMA 2015;313:853-5.

48. Maisonneuve P, Bagnardi V, Bellomi M, et al. Lung cancer risk prediction to select smokers for screening CT--a model based on the Italian COSMOS trial. Cancer Prev Res (Phila) 2011;4:1778-89.

49. Kovalchik SA, Tammemagi M, Berg CD, et al. Targeting of low-dose CT screening according to the risk of lungcancer death. N Engl J Med 2013;369:245-54.

50. de-Torres JP, Wilson DO, Sanchez-Salcedo P, et al. Lung cancer in patients with chronic obstructive pulmonary disease. Development and validation of the COPD Lung Cancer Screening Score. Am J Respir Crit Care Med 2015;191:285-91.

51. de-Torres JP, Marín JM, Casanova C, et al. Identification of COPD patients at high risk for lung cancer mortality using the COPD-LUCSS-DLCO. Chest 2016;149:936-42.

52. Gould MK. Clinical practice. Lung-cancer screening with low-dose computed tomography. N Engl J Med 2014;371:1813-20.

53. Bach PB, Mirkin JN, Oliver TK, et al. Benefits and harms of CT screening for lung cancer: a systematic review. JAMA 2012;307:2418-29.

54. de-Torres JP, Casanova C, Marín JM, et al. Exploring the impact of screening with low-dose CT on lung cancer mortality in mild to moderate COPD patients: a pilot study. Respir Med 2013;107:702-7.
55. Wille MM, Dirksen A, Ashraf H, et al. Results of the Randomized Danish Lung Cancer Screening Trial with Focus on High-Risk Profiling. Am J Respir Crit Care Med 2016;193:542-51.

56. Young RP, Duan F, Chiles C, et al. Airflow Limitation and Histology Shift in the National Lung Screening Trial. The NLST-ACRIN Cohort Substudy. Am J Respir Crit Care Med 2015;192:1060-7.

57. Choong CK, Meyers BF, Battafarano RJ, et al. Lung cancer resection combined with lung volume reduction in patients with severe emphysema. J Thorac Cardiovasc Surg 2004;127:1323-31.

58. Wang W, Xu Z, Xiong X, et al. Video-assisted thoracoscopic lobectomy for non-small cell lung cancer in patients with severe chronic obstructive pulmonary disease. J Thorac Dis 2013;5 Suppl 3:S253-9.

59. Vaughan P, Oey I, Nakas A, et al. Is there a role for therapeutic lobectomy for emphysema? Eur J Cardiothorac Surg 2007;31:486-90; discussion 490.

60. Makey I, Berger RL, Cabral HJ, et al. Maximal Oxygen Uptake-Risk Predictor of NSCLC Resection in Patients With Comorbid Emphysema: Lessons From NETT. Semin Thorac Cardiovasc Surg 2015;27:225-31.

61. Choong CK, Mahesh B, Patterson GA, et al. Concomitant lung cancer resection and lung volume reduction surgery. Thorac Surg Clin 2009;19:209-16.

62. Zhang Y, Sun Y, Wang R, et al. Meta-analysis of lobectomy, segmentectomy, and wedge resection for stage I non-small cell lung cancer. J Surg Oncol 2015;111:334-40.

63. Shirvani SM, Jiang J, Chang JY, et al. Lobectomy, sublobar resection, and stereotactic ablative radiotherapy for earlystage non-small cell lung cancers in the elderly. JAMA Surg 2014;149:1244-53.

64. Fiorelli A, Costanzo S, di Costanzo E, et al. The early detection of lung cancer during follow-up of patients undergoing endobronchial one-way valve treatment for emphysema. Arch Bronconeumol 2015;51:e13-5.

65. Westover KD, Seco J, Adams JA, et al. Proton SBRT for medically inoperable stage I NSCLC. J Thorac Oncol 2012;7:1021-5.

66. Palma D, Lagerwaard F, Rodrigues G, et al. Curative treatment of Stage I non-small-cell lung cancer in patients with severe COPD: stereotactic radiotherapy outcomes and systematic review. Int J Radiat Oncol Biol Phys 2012;82:1149-56.

67. Ashraf H, Saghir Z, Dirksen A, et al. Smoking habits in the randomised Danish Lung Cancer Screening Trial 
with low-dose CT: final results after a 5-year screening programme. Thorax 2014;69:574-9.

68. Slatore CG, Baumann C, Pappas M, et al. Smoking behaviors among patients receiving computed tomography for lung cancer screening. Systematic review in support of the U.S. preventive services task force. Ann Am Thorac Soc 2014;11:619-27.

69. Tønnesen P. Smoking cessation and COPD. Eur Respir Rev 2013;22:37-43.

Cite this article as: Gonzalez J, Marín M, Sánchez-Salcedo $\mathrm{P}$, Zulueta JJ. Lung cancer screening in patients with chronic obstructive pulmonary disease. Ann Transl Med 2016;4(8):160. doi: 10.21037/atm.2016.03.57 